\title{
Connection tubing causing small bowel obstruction and colonic erosion as a rare complication after laparoscopic gastric banding: a case report
}

\author{
Liza BK Tan*, Jimmy BY So and Asim Shabbir
}

\begin{abstract}
Introduction: Laparoscopic adjustable gastric banding is the most frequently performed bariatric procedure for the treatment of morbid obesity and is associated with low morbidity and mortality. Complications related to obesity surgery are rare and their presentation is often non-specific. Thus, it is highly important for physicians who are practising bariatric surgery to be aware of complications described in single-case studies or series when they come across similar complications even years after the primary bariatric operation.
\end{abstract}

Case presentation: We report the case of a 47-year-old Malay woman who was admitted with symptoms and signs suggesting intestinal obstruction five years after gastric band placement.

Conclusions: In our patient, the band connection wire tube was the cause of both small bowel obstruction and colonic erosion. Computed axial tomography is the cornerstone of the investigation of such patients. After surgical removal of the connecting tube, our patient recovered without sequelae.

\section{Introduction}

In this era of rising body mass indices, the need for bariatric surgery also is on the rise. Laparoscopic adjustable gastric banding (LAGB) is the most frequently performed bariatric procedure for the treatment of morbid obesity and is considered the least invasive form of bariatric surgery. LAGB, compared with other bariatric procedures, results in a shorter hospital stay, faster recovery, and cosmetically ideal scars. In addition, it is the only form of obesity surgery that is reversible. The long-term weight loss is approximately $50 \%$ of excess weight [1-4]. LAGB is associated with low morbidity and mortality. In the literature, the overall complication rate is reported to be between $9 \%$ and $13 \%$. We report a rare late complication: a simultaneous colonic erosion and small bowel obstruction caused by a silicone connecting tube five years after an LAGB.

\section{Case presentation}

A 47-year-old Malay woman presented with a one-day history of symptoms suggestive of intestinal obstruction,

\footnotetext{
* Correspondence: liza_bk_tan@nuhs.edu.sg

National University Hospital, 5 Lower Kent Ridge Road, Singapore 119074
}

which included severe colicky abdominal pain and profuse vomiting. She had had an LAGB placement five years before. Her initial body mass index had been 59.1. Seven months after placement of the LAGB, the procedure had been complicated by a band connection wire tube leak and a port site dislocation, which necessitated a resetting of the reservoir and a trimming of the tubing. After this procedure, she was subsequently lost to follow-up. When she presented to our hospital, her body mass index was 28.2 .

During a physical examination, she was noted to have abdominal distention and tenderness. The results of her laboratory test on admission showed a white blood cell count of $8.07 \times 109 / \mathrm{L}$. An X-ray of the abdomen revealed a loop of dilated small bowel in the upper abdomen, and air and fluid levels.

The stomach bubble was prominent. On a computed axial tomography scan (Figure 1), small bowel and stomach were noted to be dilated. The connection tubing appeared to be looping around the mesentery of the small bowel. A gastroscopy (Figure 2) demonstrated gastric band erosion of the lesser curve.

Our patient proceeded to the operating room. During the operation, the proximal small bowel and mid-

\section{C) Biomed Central}

(C) 2012 Tan et al; licensee BioMed Central Ltd. This is an Open Access article distributed under the terms of the Creative Commons Attribution License (http://creativecommons.org/licenses/by/2.0), which permits unrestricted use, distribution, and reproduction in any medium, provided the original work is properly cited. 

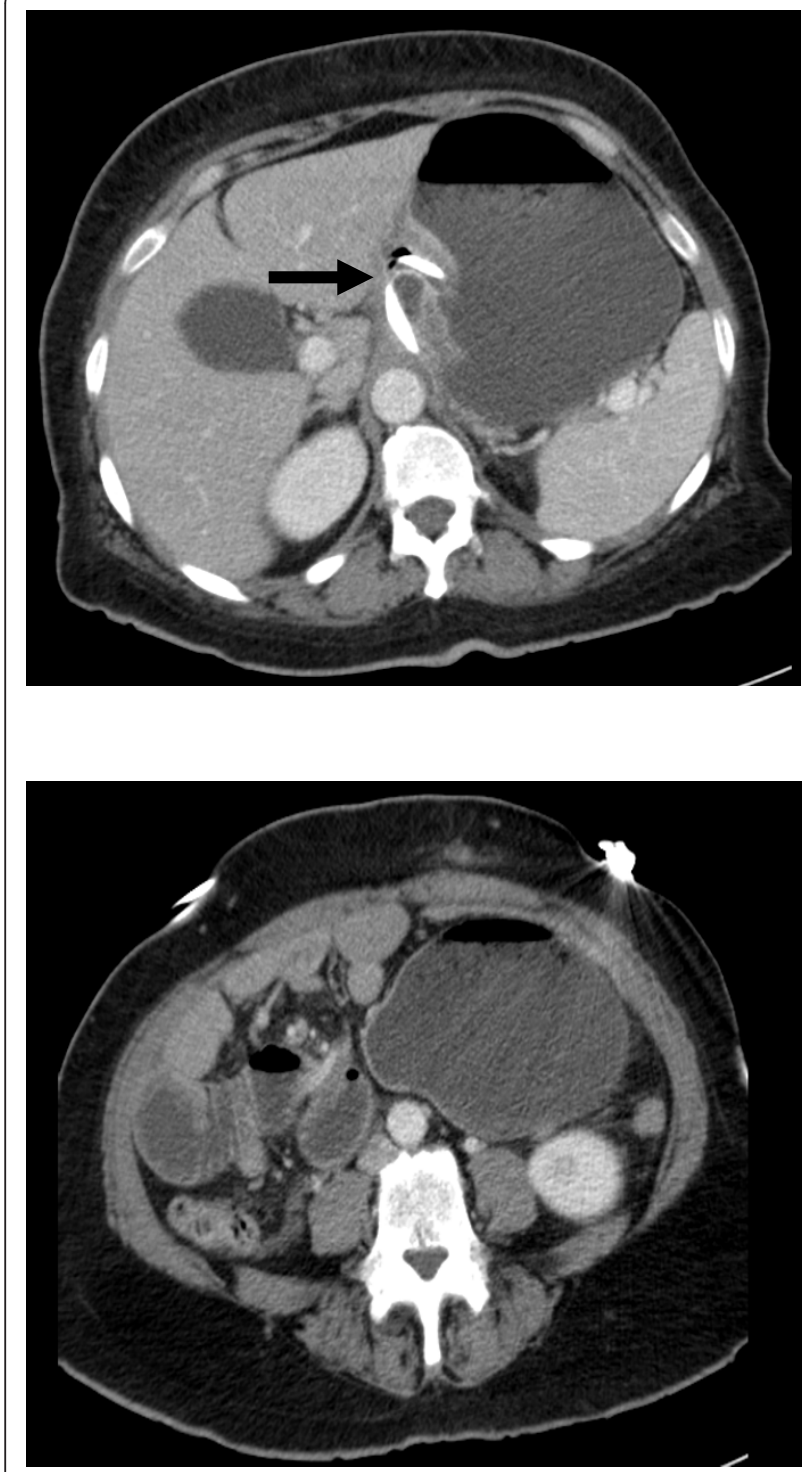

Figure 1 A computed axial tomography image of the dilated stomach with a band in position. Connection tubing can be seen wrapping around the dilated small bowel (arrow).

transverse colon were densely adherent to tubing. The surgeons were unable to dissect this safely, and a decision was made to convert to a full laparotomy. During the laparotomy, the adhesions were taken down sharply to reveal the tubing sandwiched between the small bowel anterior and the mid-transverse colon posteriorly (Figure 3a). It was noted, on closer inspection, that the tubing had eroded into the colon (Figure $3 \mathrm{~b}$ ). The tubing was dissected away from the mesentery and freed the small bowel, and the colonic perforation was closed with, primarily, 3.0 polydioxanone sutures in two layers. The gastric band was removed, and the lesser curve of the stomach was repaired with vicryl

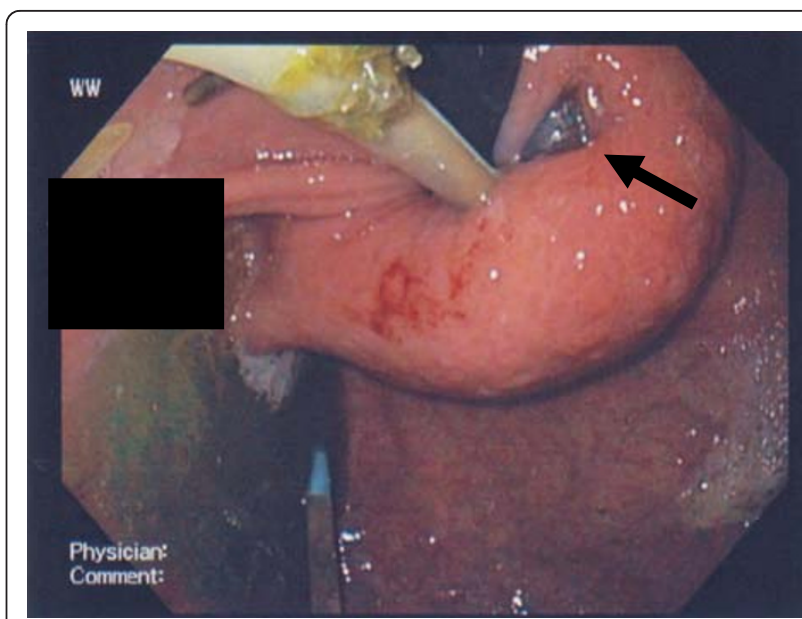

Figure $2 \mathrm{~A}$ retroflexion view of a nasogastric tube passing through the gastroesophageal junction during a gastroscopy and a part of the band eroding though the mucosa.

2.0 sutures. A tongue of omentum was secured over the repair.

Our patient's recovery was uneventful. A gastrografin study was carried out on postoperative day five. After the absence of a contrast leak was confirmed, our patient went on a diet and was discharged on postoperative day eight. Three months after discharge, our patient had gained 15 pounds.

\section{Discussion}

In the literature, the overall complication rate of LAGB is reported to be between $9 \%$ and $13 \%$. Complications are generally related to the band or band connection wire tubing or the port. Band-related complications are well described in the literature.

They include the more commonly described gastric erosion ( $0 \%$ to $11 \%)$, pouch dilatation (5\% to $17 \%)$, slippage (1\% to $5 \%)$, migration (1\%), and, rarely, gastric perforation (0\% to 3\%) [1-4]. Himpens and colleagues [5] reported that, in a 12-year period, nearly one in three patients experienced band erosions.

Although intragastric migration of adjustable bands has been widely described, colonic erosion is extremely rare and has been described only in small case series [6,7]. Similarly, case studies have described band connection wire tubing constriction around small bowel [8-12] or cecum [8] causing bowel obstruction. In one case study, a patient had severe diffuse abdominal pain in addition to port site pain as a result of traction by the band connection wire tube around the small bowel mesentery, pulling the mesentery into the right iliac fossa [9].

Interestingly, in a systemic review of band erosions after LAGB [10], a multiple regression analysis showed that the erosion rate is significantly predicted by the 


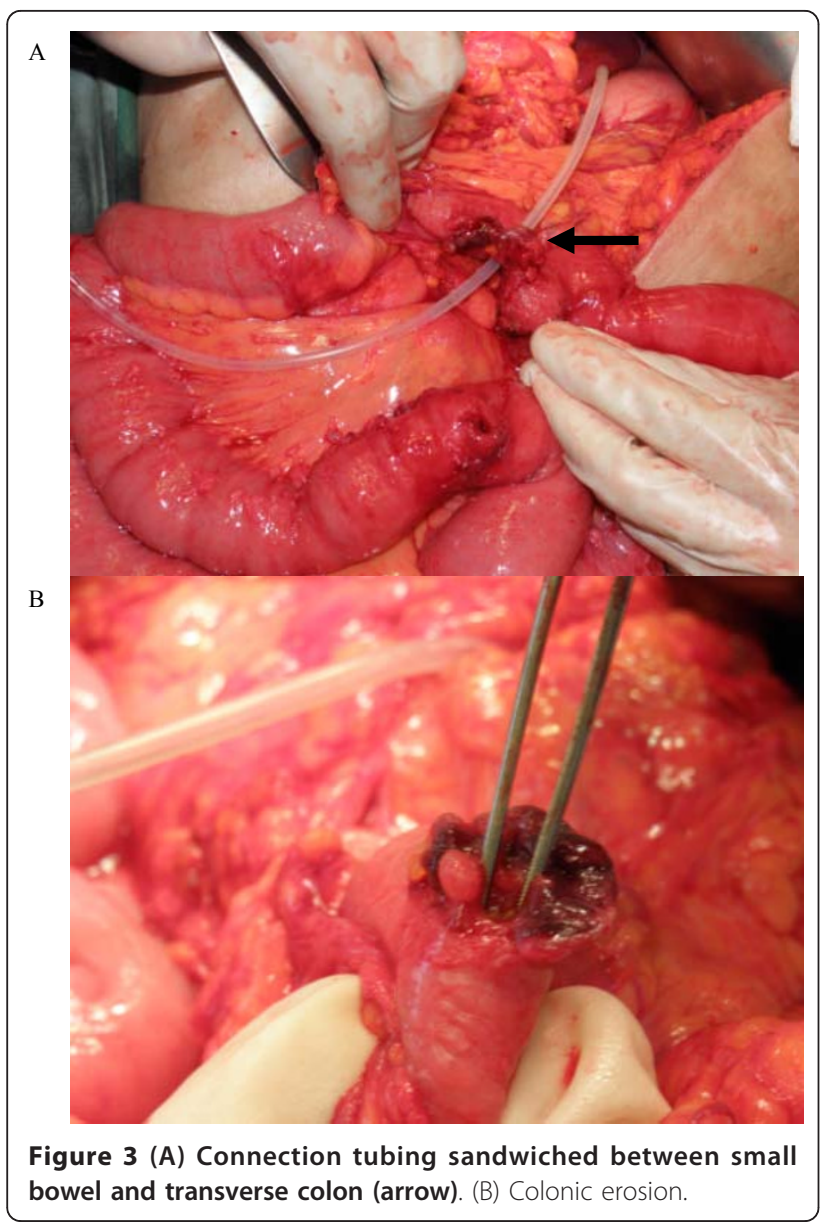

experience of the bariatric surgeon. Port site placement and length of band connection wire tubing have not been shown to be directly related to these complications. Intuitively, it appears that altering these factors may help in reducing these complications, but the exact method is still obscured.

\section{Conclusions}

To the best of our knowledge, this is the first report of simultaneous gastric erosion, colonic erosion, and small bowel obstruction following gastric banding. Each complication is reported separately in case reports and case series only. These complications have not simultaneously occurred in any one patient. In this case report, we demonstrate that various combinations of complications can occur in a bariatric patient many years after the original procedure and that the cornerstone of the evaluation of bowel obstruction in these patients remains the computed axial tomography scan [13]. We have already mentioned the fact that complications of bariatric surgery remain rare and are non-specific; therefore, through the reporting of complications, we may be able to share our experience with other bariatric surgeons.

\section{Consent}

Written informed consent was obtained from the patient for publication of this case report and any accompanying images. A copy of the written consent is available for review by the Editor-in-Chief of this journal.

\section{Abbreviation}

LAGB: laparoscopic adjustable gastric banding.

\section{Authors' contributions}

LBKT reviewed the case and prepared the manuscript. AS was the primary physician of this patient. He reviewed and treated this patient in conjunction with JBYS. Both AS and JBYS were major contributors in the writing of the manuscript. All authors read and approved the final manuscript.

\section{Competing interests}

The authors declare that they have no competing interests.

Received: 21 April 2011 Accepted: 11 January 2012

Published: 11 January 2012

\section{References}

1. Doldi SB, Micheletto G, Lattuada E, Zappa MA, Bona D, Sonvico U: Adjustable gastric banding: 5-year experience. Obes Surg 2000, 10:171-173.

2. Wolf AM, Kortner B, Kuhlmann HW: Results of bariatric surgery. Int J Obes Relat Metab Disord 2001, 25:S113-114.

3. Coskun H, Bozbora A, Ogunc G, Peker Y: Adjustable gastric banding in a multicenter study in Turkey. Obes Surg 2003, 13:294-296.

4. Weiner R, Blanco-Engert R, Weiner S, Matkowitz R, Schaefer L, Pomhoff I: Outcome after laparoscopic adjustable gastric banding- 8 years experience. Obes Surg 2003, 13:427-434

5. Himpens J, Cadière GB, Bazi M, Vouche M, Cadière B, Dapri G: Long-term outcomes of laparoscopic adjustable gastric banding. Arch Surg 2011, 146:802-807.

6. Zappa MA, Lattuada E, Mozzi E, Francese M, Antonini I, Radaelli S, Roviaro G: An unusual complication of gastric banding; recurrent small bowel obstruction caused by the connecting tube. Obes Surg 2006, 16:393-341.

7. Shipkov CD, Uchikov AP, Uchikova EH: Small bowel obstruction by the silicone tube of the gastric band. Obes Surg 2004, 14:1280-1282.

8. Strobos E, Antanavicius G, Josloff R: Unusual complication: small bowel obstruction caused by tubing of gastric band. Surg Obes Relat Dis 2009, 5:697-640.

9. Fass G, Simoens C, Mendes da Costa P: Diffuse abdominal and port site pain caused by the connecting tube in gastric banding. Obes Surg 2008, 20:1183-1185.

10. Egbert K, Brown WA, O'Brien PE: Systemic review of erosion after laparoscopic adjustable gastric banding. Obes Surg 2011, 21:1272-1279.

11. Van de Water W, Vogelaar FJ, Willems JM: An unusual complication 4 years after laparoscopic adjustable banding; jejunal obstruction due to the connecting tube. Obes Surg 2009, 21:131-133.

12. Agahi A, Harle R: A serious but rare complication of laparoscopic adjustable gastric banding: bowel obstruction due to caecal volvulus. Obes Surg 2008, 19:1197-1200.

13. Stoker J, van Randen A, Laméris W, Boermeester MA: Imaging patients with acute abdominal pain. Radiology 2009, 253:31-46.

\section{doi:10.1186/1752-1947-6-9}

Cite this article as: Tan et al: Connection tubing causing small bowel obstruction and colonic erosion as a rare complication after laparoscopic gastric banding: a case report. Journal of Medical Case Reports 2012 6:9. 